\title{
J. Passon: Shakespeare in der Realschule inszenieren. Theatre Education zur Förderung von Kommunikativer und Performativer
} Fremdsprachenkompetenz

Reihe: Fremdsprachendidaktik in globaler Perspektive - Bd. 3, 2014, 296 S., 29.90 EUR, 47.90 CHF, br., ISBN

978-3-643-12801-0

Stefanie Beckmann

Die in Buchrezensionen vertretenen Ansichten und Meinungen sind die der jeweiligen Rezensentinnen und Rezensenten und reflektieren nicht notwendigerweise die Position von SCENARIO.

$\S$

Bei diesem Band handelt es sich um eine empirische Studie, deren Ziel es ist darzulegen, dass die von der Autorin formulierte English Theatre Class-Theorie (ETC) die kommunikative und performative Fremdsprachenkompetenz von Mittelstufenschülerinnen und -schülern fördert und verbessert. In acht Kapiteln beschreibt Passon das Design ihrer außercurricularen englischsprachigen Arbeitsgemeinschaft (English Theatre Class) an einer Realschule in BadenWürttemberg sowie die in diesem Rahmen vorgenommene Aktionsforschung mit Hilfe unterschiedlicher Datenquellen. Ein Online-Appendix mit Transkriptionen und Beobachtungsnotizen findet sich auf der Webseite des LIT-Verlags. Vorläufer dieser Datenerhebung sind Erkenntnisse aus einer explorativen Studie (Passon 2008) und einer Pilotstudie (Passon 2011).

Nach einer Einleitung (Kapitel 1) gibt Passon im zweiten Kapitel („Das Untersuchungsfeld Fremdsprachenunterricht in der Realschule") einen Abriss über den aktuellen Stand der Fremdsprachendidaktik in der Sekundarstufe 1. Ihr zufolge ist zwar ein Trend zu drama- bzw. theaterpädagogischen Mitteln zu erkennen, aber eine performative Kompetenz als kommunikative Fertigund Fähigkeit (skill) sei im Gemeinsamen Europäischen Referenzrahmen für Sprachen und den Bildungsstandards Baden-Württembergs bisher nicht formuliert.

Im dritten Kapitel („Shakespeare und Fremdsprachenunterricht") beleuchtet Passon die Relevanz von Shakespeares Werken im Fremdsprachenunterricht und legt dar, dass Realschüler mit literarischen Texten generell und mit 
Shakespeare im Besonderen wenig in Kontakt kommen. Sie plädiert für einen Einsatz Shakespeares in der Realschule im Sinne eines dramatic approach, der eine analytische Herangehensweise an Romeo and Juliet ausblendet und stattdessen „eine doppelte Perspektive Hinwendung zum Text und zu den Reaktionen auf den Text" erlaubt (40).

Im vierten Kapitel („Theatre education und Fremdsprachenkompetenzen“) versucht Passon eine Definition und Abgrenzung von drama education, theatre education, drama in education und theatre in education, um ihr methodisches Vorgehen und ihre ETC-Theorie zu erklären. Sie folgert: „Daraus ergibt sich zunächst, dass zwischen drama in education und theatre education insofern unterschieden wird, als dass mittels theatre education eine theaterästhetische Differenzerfahrung gefördert werden soll. Aus dieser Annahme heraus wird die ETC-Theorie hergeleitet, die - so meine These - in der praktischen Umsetzung durch eine englischsprachige Theater-Arbeitsgemeinschaft (AG) auch zur Förderung von Fremdsprachenkompetenzen führen kann." (4) Es ist dieses „kann“, das Passons' Studie durchgehend begleitet und relativiert (siehe weitere Ausführungen).

Passon erläutert, dass sie Theater als autonomes Mittel im Sinne einer Ermöglichungspädagogik nach Martens (2008) nutze, in der „die Förderung von Fremdsprachenkompetenzen eine sekundäre Rolle spielt" (249), aber positive Effekte auf diese anerkenne. Sie führt aus, dass es im Gegensatz zu anderen Bundesländern - in Baden-Württemberg kein theateraffines Fach in der Sekundarstufe 1 gebe und schließt daraus, dass „theatre education [...] nicht im Regelunterricht gefördert werde. Es müssten folglich Alternativen gefunden werden, um den Austausch von Theaterpädagogik und Fremdsprache - mit dem Ziel einer kreativ-schöpferischen Arbeit - zu ermöglichen“ (44).

Ihr Unterrichtsdesign der Arbeitsgemeinschaft benutzt den gekürzten Originaltext, der adaptiert wird in eine moderne Fassung mit Bezug zur Lebenswelt der Schülerinnen und Schüler - Romeo and Juliet - a local football version -, die nach sechsmonatiger Probenarbeit zur Aufführung gebracht wird. Sie erläutert nachvollziehbar die Relevanz der Shakespearischen Themen für heutige Jugendliche (Schnittmenge von Rollenträger und Rollenfigur nach Ruping 2011) warum allerdings im lokal verorteten Stück Romeo nach Mantua verbannt wird, bleibt unverständlich (113) -, um im Anschluss das methodisch-didaktische Vorgehen im ETC-Unterricht zu schildern, in dem die Lerner als Schauspieler behandelt werden und der folgenden didaktischen Aufbau umfasst: theatre training, storytelling, role-taking, scene work, scene performance, dress rehearsal, performance nights, reflection. Die Unterrichtsstruktur besteht aus warm up, scene work, scene performance, cool down.

Der Unterricht findet in englischer Sprache statt, wobei Passon betont: „Die Besonderheit der ETC-Arbeit ist jedoch, dass der Fokus gerade nicht auf der Sprachvermittlung liegt.“ (64). Um feststellen zu können, ob und inwieweit die kommunikative Fremdsprachenkompetenz in diesem Ansatz gefördert werden kann, argumentiert Passon für eine Eingrenzung des kommunikativen und performativen Kompetenzbegriffs und folgt in ihrer Studie zur Analyse 
der kommunikativen Kompetenz weitgehend dem Modell von Bachman und Palmer (1996) und einem performativen Kompetenzbegriff, der auf den Kriterien 'Wissen über Inszenierungen,' 'Erkennen künstlerischer Qualität' und 'Selbstvertrauen' basiert .

Im fünften Kapitel („Das Forschungsprojekt Shakespeare in der Realschule inszenieren") erläutert die Autorin die quantitativen und qualitativen Datenerhebungs- und Auswertungsmethoden unter Beteiligung der Perspektiven der Autorin selbst, vier anderer Lehrpersonen und 18 Schülerinnen und Schüler der Jahrgänge 8 und 9. Passon sammelt Daten in Form von informellen Beobachtungsnotizen, Fragebögen zu unterschiedlichen Zeitpunkten innerhalb des Projekts, Fallanalysen, Interviews, und Videosequenzen und räumt ein: „Insgesamt ist die Studie hauptsächlich im qualitativen Paradigma anzusiedeln, die die Sichtweise der Schüler und der forschenden Lehrkraft in den Mittelpunkt stellt." (4). Sie verzichtet auf Zielformulierungen, Hypothesen und Indikatoren, aber formuliert folgende vier Forschungsfragen: „1. Welche Auswirkungen hat theatre education auf die kommunikative Fremdsprachenkompetenz der teilnehmenden Realschüler? 2. Welche Auswirkungen hat theatre education auf die performative Fremdsprachenkompetenz der teilnehmenden Realschüler? 3. Können kommunikative und performative Fremdsprachenkompetenz an der Realschule auf der Basis der ETC-Theorie entwickelt werden? Wie wirkt sich die Arbeit am Shakespeare-Text basierend auf der ETC-Theorie auf die persönliche Haltung der Schüler aus, Englisch zu lernen?" (79). Die Videosequenzen basieren inhaltlich auf der Improvisationsübung bus stop von Kurtz (2001), die zu zwei Zeitpunkten im Laufe des Projekts aufgezeichnet und durch externe Lehrkräfte mit Hilfe eines Bewertungsbogens in Anlehnung an den 'Test of Spoken English' (126f) ausgewertet wurden.

Im sechsten Kapitel („Datenanalyse und Ergebnisse“) wertet Passon die einzelnen Ergebnisse ihrer Studie hinsichtlich des Mehrwerts des Projekts für die Schülerinnen und Schüler aus und inwiefern es „einen subjektiven Lernzuwachs“ (5) befördert. Sie räumt ein, dass nicht alle erhobenen Daten verwertbar seien. Passon listet die Mittelwerte aus Pre- und Post-Fragebogen und den Grad der Veränderung im Regelunterricht. In den Lehrerfragebögen überschreiten die Veränderungen den Wert von +0,4 nicht, von schwachen positiven Veränderungen im in der Kategorie Verstehen (Erschließungsstrategien) bis zu etwas stärkeren positiven Veränderungen in den Kategorien Performanz (Selbstvertrauen), Haltung, Sprechen (Flüssigkeit), Verstehen und Sprechen (Wortschatz). Erstaunlich sind im Vergleich die Ergebnisse der Schülerfragebögen, die mehrfach negative Veränderungen im Verhalten im Regelunterricht aufweisen.

Im siebten Kapitel („Diskussion der Ergebnisse“) diskutiert Passon die Antworten auf die anfangs gestellten vier Forschungsfragen (siehe oben). Aus ihrer Sicht als teacher-researcher, v.a. anhand der von ihr ausgewählten Fallanalysen, „kann von einer starken positiven Auswirkung von theatre education auf die allgemeine kommunikative Fremdsprachenkompetenz ausgegangen werden“ (239). Sie wertet die Ergebnisse der Triangulation der Daten aus und 
beantwortet alle vier Untersuchungsfragen positiv.

Im achten Kapitel („Zusammenfassung und Ausblick“) fasst Passon die vorangegangenen Kapitel zusammen und wagt dann auf fünf Seiten einen Ausblick auf mögliche fachdidaktische Konsequenzen ihrer Arbeit. Sie stellt zunächst fest, dass das "Themenfeld performative Fremdsprachenkompetenz" (251) noch zu wenig erforscht sei und ein Kompetenzmodell samt Operationalisierung noch ausstehe. Angelehnt an Hallet (2010) und Bachman und Palmer (1996) entwirft sie ein Modell, das einige ihrer Forschungsbereiche (Kategorie 4: Selbstvertrauen, Kategorie 5: Erkennen der künstlerischen Qualität, Kategorie 6: Wissen über Inszenierungen, Kategorie 7: Haltung) integriert, aber zu den für den Fremdsprachenunterricht relevanten Kategorien Verstehen (K1), Verstehen und Sprechen (K2), Sprechen (K3) keine differenzierten Aussagen macht. Sie plädiert dafür, die ausgewiesene Kompetenz Sprechen in den Bildungsplänen die Komponente "performative Kompetenz" hinzuzufügen und macht Vorschläge zur Erweiterung des Gemeinsamen Europäischen Referenzrahmens für Sprachen: „Die Schülerinnen und Schüler (SuS) können Szenen über vertraute [...] Themen ästhetisch-expressiv darstellen, im Rahmen von inszenierten Wirklichkeiten ihre Meinung ausdrücken und Informationen austauschen" (254).

Folgende Aspekte sind in Passons Arbeit kritisch zu sehen:

- Passons Recherche zur Verbreitung von theatre education mit den unterschiedlichen Schulfachbezeichnungen wie Darstellendes Spiel, Darstellen und Gestalten oder Kultur und Künste in den einzelnen Bundesländern ist in Zahlen zu niedrig. Das Land Nordrhein-Westfalen fehlt in ihrer Listung (44).

- Passon folgt in ihrem AG-Design dem üblichen theaterpädagogischen Vorgehen und betont folgerichtig die notwendige theaterpädagogische Vorbildung der Fremdsprachenlehrkraft. Eine eigene Theorie (die von ihr genannte ETC-Theorie) lässt sich hinter diesem Ansatz auch mit viel Wohlwollen nicht erkennen.

- Den Ausführungen der Autorin zufolge liegt ihr Schwerpunkt auf dem Ansatz der theatre education; soziale Kompetenz werde demnach nicht berücksichtigt. Dies bleibt unverständlich, denn Theaterarbeit ist Interaktion, ein Zusammenspiel von Aktion und Reaktion, das Aufnehmen und Umwandeln von Impulsen.

- Warum die Autorin sich weigert, aktive Förderung und Erweiterung von Wortschatzspektrum durchzuführen, bleibt unverständlich. Gerade, wenn es darum gehen soll, die ästhetischen Momente im Sinne einer performativen Kompetenz zu unterstreichen, die Schülerinnen und Schüler zur selbstständigen Erarbeitung von Szenen anzuleiten und die Szenen auszuwerten, ist ein Fachjargon, der zum Beispiel Bewegungsarten oder Sprechweisen beschreibt, unerlässlich. Von ausgesuchten Fachtermini 
dieser Art profitieren die Schülerinnen und Schüler auch im Regelunterricht (z.B. in Bildbeschreibungen, Charakteranalysen, im kreativen Schreiben, in Planung und Auswertung von Rollenspielen, ... ).

- Das Datenmaterial ist insgesamt widersprüchlich. Bei den eingesetzten Fragebögen für die Schülerinnen und Schüler fällt auf, dass sie einzelne Items Wertungen wie „flüssig“, „mühelos“, „ich glaube“ enthalten. Diese in den Items enthaltenen Beurteilungen machen die Skala redundant: „Eine neue Grammatik kann ich mühelos mündlich anwenden“ ist bereits die beste Leistung, die erreicht werden kann und macht eine Angabe von „trifft voll zu“, „trifft überwiegend zu“ etc. überflüssig. Die Skala enthält 5 Abstufungen, was mittlerweile eher unüblich ist, weil sie die Tendenz zur Mitte („trifft teilweise zu“) verstärkt (120f). Bei der Bewertung der Videosequenzen wird deutlich, dass sich die Einschätzungen der externen Lehrer erheblich unterscheiden (153). So wäre es sicherlich sinnvoll gewesen, für die Evaluation bzw. Messung der Progression zur Verfügung stehende externe Instrumente wie die landesspezifischen Vergleichsarbeiten zu nutzen oder die Informantenzahl zu erhöhen. Zum Abschluss des Projekts führte Passon 15-minütige Befragungen in Form eines Leitfrageninterviews mit jeweils zwei Schülerinnen und Schülern durch. Die Fragen erscheinen, trotz Gegenrede der Autorin, suggestiv, z.B. „Seht ihr darin Vorteile, eine Fremdsprache auch durch ein englischsprachiges Theaterstück zu lernen? Wenn ja, warum?“, „Ist ETC hilfreich, um das Sprechen vor einem Publikum zu [Wort fehlt]. Wenn ja, warum?" (130).

- Beim Vergleich der Bewertung der Videosequenzen durch beobachtende Lehrpersonen und der Schülerantworten in den Interviews fallen immer wieder Diskrepanzen auf, die von Passon nicht zufriedenstellend geklärt werden. So meint zum Beispiel S1 (Schülerin 1), dass sie ,jetzt im Englischunterricht wesentlich mehr verstehe als vor ETC“ (155). Sie gibt an, dass sie nun mehr Wörter kenne und mehr auf Englisch sagen könne. In den Übungen bus stop hingegen wurde von den beobachten Lehrkräften keine positive Veränderung festgestellt. Ähnlich verhält es sich beim Vergleich der Fallanalysen mit den anderen Daten. Zum Beispiel wird S4 der Klassenstufe 8 von der Autorin als eine Schülerin mit sehr guten Leistungen genannt, aber S8 als Beispiel für eine Schülerin der Klassenstufe 8 mit guten bis befriedigenden Leistungen wird in beiden Videosequenzen wesentlich besser bewertet als S4. Als Fallanalysen hätten S1 (keine Veränderung der kommunikativen Leistungen der Schüler während ETC per Videobewertung), S3 (sehr starke positive Veränderung der kommunikativen Leistungen der Schüler während ETC per Videobewertung) und S14 (negative Veränderung der kommunikativen Leistungen der Schüler während ETC per Videobewertung) sicherlich mehr interessiert als die von Passon ausgewählten. In den Beobachtungsnotizen der zweiten Woche schreibt Passon, dass S3 sie durch 
deren ihr unbekannte Lebhaftigkeit während bus stop erstaune, in der Bewertung durch die externen Lehrkräfte schneidet die Schülerin aber mit einem Mittelwert gegen 0 ab. Über S10 schreibt Passon: „[. . . ] ist sehr bemüht. Seine fremdsprachlichen Grenzen werden trotz seiner deutschen Redseligkeit deutlich“ (199). Dieser Schüler liegt in der Bewertung der externen Lehrkräfte im oberen Drittel der Gruppe.

- Eine Umgestaltung des GER wurde bereits von Fonio \& Genicot (Scenario 2/2011) vorgeschlagen. In den konkreten Umsetzungen des GER bzw. den Bildungsplänen der einzelnen Bundesländer finden sich Ansätze, die den Vorschlägen der Autorin entgegenkommen. Zum Beispiel Im Kernlehrplan Englisch der Realschule NRW (http://www.schulentwicklung.nrw.de) finden sich folgende Vorgaben: „Sprechen: an Gesprächen teilnehmen. Die Schülerinnen und Schüler können sich in einfachen themenorientierten Gesprächssituationen des Unterrichts sowie in Grundsituationen des Alltags verständigen, vorausgesetzt sie sind hierauf sprachlich vorbereitet. Sie können [... ] in Rollenspielen Perspektiven unterschiedlicher Personen vortragen.“ und „Handeln in Begegnungssituationen. Die Schülerinnen und Schüler können in fiktiven und realen Begegnungssituationen im eigenen Umfeld und auf Reisen ihr Repertoire an kulturspezifischen Verhaltensweisen (z. B. Höflichkeitsformeln, Modalverben beim meinungsbetonten Sprechen) anwenden und erweitern." Interessant wären an den Lehrplänen konkret ausgerichtete Vorschläge zur performativen Kompetenz, die auf die sprachlichen Vorgaben bzw. zu erreichenden Kompetenzstufen differenziert Bezug nehmen. Ein Blick über die eigenen Landesgrenzen hinaus und auf das, was Lehrpersonen in ihrem Unterricht tatsächlich tun, erscheint für eine mögliche weitere Forschungsarbeit der Autorin durchaus lohnenswert.

- Eine Untersuchung, die die kommunikativen Kompetenzen im Regelunterricht misst und in der Folgezeit (nach der Teilnahme an ETC) eine Stabilisierung der positiven Effekte durch ETC zeigt, steht aus.

In dieses Buch ist offensichtlich viel Arbeit geflossen. Nichtsdestoweniger haftet Passons Werk eine Unentschiedenheit an. Sie möchte zeigen, dass Shakespeare in der Realschule funktioniert - das glauben wir ihr gerne - und dass die Schülerinnen und Schüler einen Mehrwert durch die Beschäftigung mit Shakespeare erfahren. Diesen Beweis erbringt Passon allerdings nicht und bietet darüber hinaus auch zu wenig konkrete Hilfen an. Der Leser sucht vergebens nach den genannten Rollenkarten, Redemitteln, Empfehlungen für vereinfachte, gut einsetzbare Textversionen etc. Weiterhin hört diese Studie an der Stelle auf, an der es eigentlich interessant wird und der Forschung bedarf. Dass Drama und Theater sowohl muttersprachliche als auch fremdsprachliche Schülerinnen und Schüler in vielerlei Hinsicht fordern und fördern, ihnen andere Fähigkeiten und Fertigkeiten, Lernzugänge und Bearbeitungstiefen abverlangen und bieten als der Regelunterricht, ist in der Literatur hinreichend diskutiert 
und gezeigt worden. Es fehlt, wie Passon selbst schreibt, eine Verankerung im Englischunterricht (256). Dieser Herausforderung hat sich die Autorin selbst nicht gestellt. Mit ihrem außercurricularen Design umgeht sie die ausstehende Formulierung und Erprobung von Kompetenzbereichen, Qualitätskriterien, Indikatoren und Vorschlägen zur Leistungsmessung performativer Kompetenz. Dies ist der Hauptkritikpunkt an dieser Arbeit. Zusätzlich plädiert Passon für eine Reform der (baden-württembergischen) Bildungsstandards und für eine neue Lehrerausbildung. All dies sind unbestritten wichtige Aspekte, die aber in ihrem Buch nicht in ausreichender Breite und Tiefe diskutiert werden (können). Das ist ganz schön „much ado“. 